\title{
Detection by paper chromatography of imidazoles, including hydantoin-5-propionic acid, in urine after histidine dosage
}

\author{
J. E. MIDDLETON \\ From the Department of Chemical Pathology, Group Pathology Laboratory, \\ General Hospital, Southampton
}

SYNOPSIS Simple paper chromatography of urine obtained in the Figlu test has been found satisfactory for determining excess excretion of the imidazole metabolites of histidine. It has been confirmed that examination for these in addition to formiminoglutamic acid is necessary for a full assessment of this test. When excretion of the latter is not abnormal in folic-acid and/or vitamin $\mathbf{B}_{12}$ deficiency, that of urocanic acid, a glycine conjugate of urocanic acid and/or imidazole propionic acid, is usually raised. An abnormally high excretion of hydantoin-5-propionic acid after histidine has also been found in these deficiencies, and its estimation may be of additional use in the evaluation of the Figlu test.

In man histidine is metabolized via urocanic acid and imidazolone propionic acid to formiminoglutamic acid. Further degradation is impaired in folic acid and/or vitamin $B_{12}$ deficiency and results in the excretion of abnormal amounts of formiminoglutamic acid after a histidine load. Bennett and Chanarin (1961, 1962), however, have demonstrated that urocanic acid forms a variable but frequently high proportion of the histidine metabolites excreted in the urine, and have therefore advocated that the urine obtained in the Figlu test should be examined for both urocanic acid and formiminoglutamic acid in order to avoid errors in diagnosis. Since their method for estimating urocanic acid is relatively specialized and time consuming, it was decided to investigate the use of simple paper chromatography for its detection. At the same time it was hoped that this method would also detect other imidazole derivatives which have been reported to be present in urine by Brown, Silva, McDonald, Snyder, and Kies (1960) and by Hubbard and Block (1962).

Brown and Kies (1959) showed that imidazolone propionic acid can alternatively be converted in man to L-hydantoin-5-propionic acid, which is not metabolized further and is excreted in the urine. It might be expected therefore that this metabolite would also be excreted in excess by patients with folic acid-vitamin- $\mathrm{B}_{12}$ deficiency following histidine, Received for publication 23 March 1965. and serve as an additional index on this defect. A further investigation was therefore undertaken to detect it and study its excretion by paper chromatography.

\section{MATERIAL AND METHODS}

An oral dose of $15 \mathrm{~g}$. L-histidine hydrochloride was given first thing in the morning to eight normal adult volunteers and three patients with simple iron-deficiency anaemia (controls), and to 40 patients after full haematological and chemical investigation judged to have folic acid-vitamin- $B_{12}$ deficiency. Two non-anaemic patients who had generalized psoriasis were also studied. Serum folic acid and vitamin- $B_{12}$ determinations were performed on all these patients. The urine passed in the following eight hours was examined. No dietary restrictions were imposed. These test urines were compared with control samples obtained from all these subjects during the same period on the previous day, and/or that passed in the hour immediately before the test. Twenty-four-hour control urine collections were also examined from four non-anaemic patients and 10 other control patients with anaemia not due to folic acidvitamin- $B_{12}$ deficiency. In most instances the validity of the timing of the multiple urine collections was checked by estimating the creatinine excretion in each specimen, using the standard AutoAnalyser method. In some cases when the hourly pre-test save appeared to be inaccurately timed, this was corrected before testing, assuming the creatine excretion to be subject to no diurnal variation. 


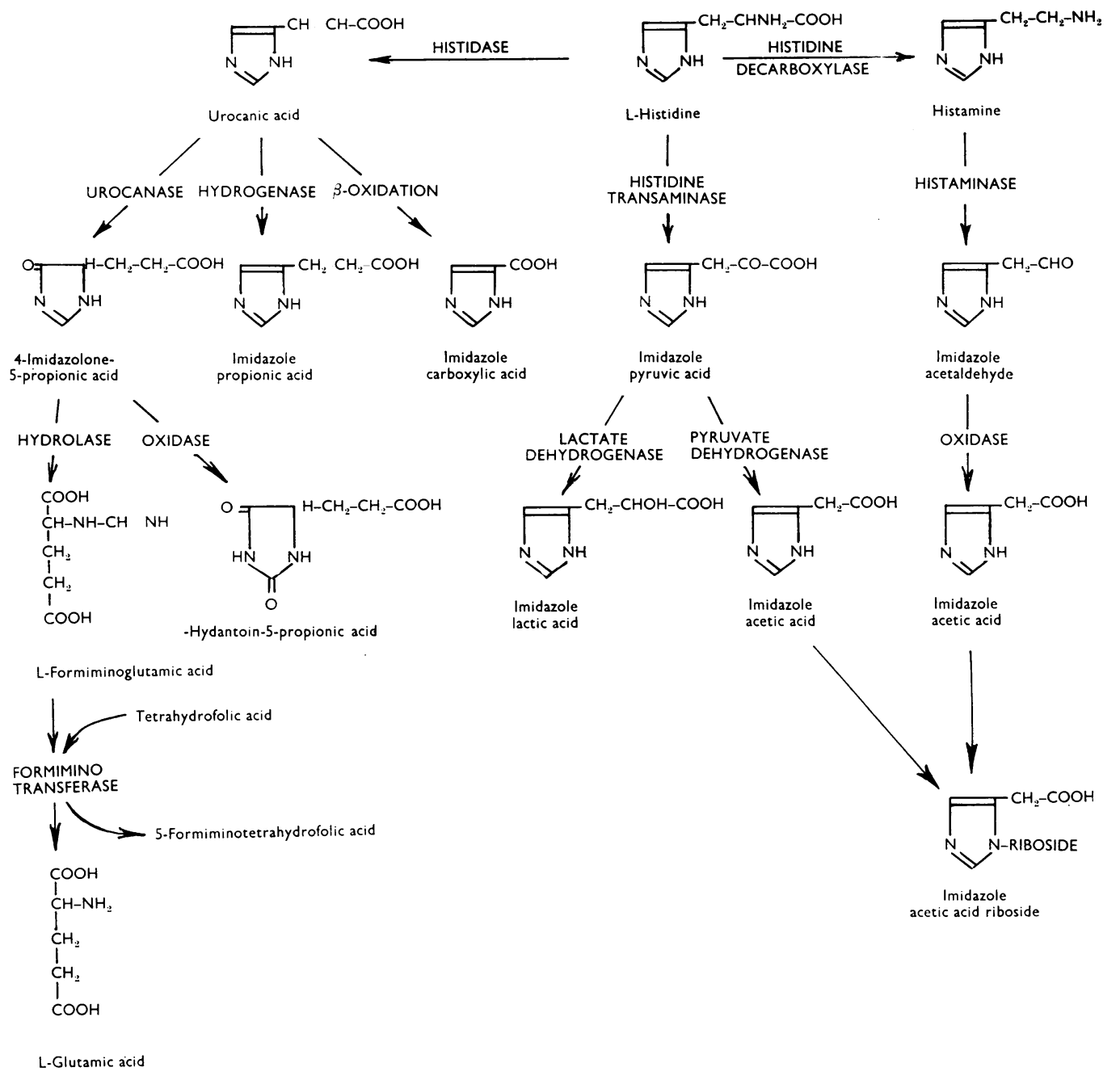

FIG. 1. Metabolic degradation of histidine

The urine was collected in bottles containing $1 \mathrm{ml}$. $6 \mathrm{~N}$ hydrochloride acid per hour of the save, and stored at $4^{\circ} \mathrm{C}$. after completion. Two-seconds urine was examined within 24 hours by two-way ascending paper chromatography on Whatman no. 1 paper using the solvents n-butanol/acetic acid/water followed by n-butanol/pyridine/water routinely, and the imidazoles were detected with Pauly's reagent as described by Smith and Birchenough (1960). Excretion was assessed semiquantitatively by observing the size and intensity of the spots. Desalting of the urine was not usually necessary, but was performed occasionally before repeat chromatography when distortion had occurred, and during further identification of the spots. This identification was performed by reference to published $\mathrm{Rf}$ values and by co-chromatography of the urine with commercially available pure compounds in the above solvents, and in iso-propanol/ammonia/water, phenol/ammonia/water, or and in n-propanol/formic acid/water. The additional $N$ methods for detecting imidazoles described by Smith 을 and Birchenough (1960) were also employed.

The test urines were also examined for formiminoglutamic acid by a modification of the paper chromatography method described by Dagnall (1962), and using a timed aliquot of urine.

For the investigation of hydantoin-5-propionic acid, two-seconds urine (or less when excretion was found to be high) was also chromatographed two ways as above, or one way using n-butanol/acetic acid/water as the solvent. Since Pauly's reagent did not detect it, the mercuric 
acetate/diphenylcarbazone dip of Bergmann and Dikstein (1958) was used; (see also McGeer, Robertson, and McGeer, 1961). This revealed a dark blue-mauve spot on a pale mauve background. Its identity in urine was confirmed by co-chromatography with pure L-hydantoin5-propionic acid in the above five solvent systems, and in benzene/n-butanol/methanol/water $(1 / 1 / 2 / 1)$ and acetic acid/n-butanol/ethyl acetate/water $(1 / 1 / 1 / 1)$. The Lhydantoin-5-propionic acid was synthesized from L-glutamic acid and potassium cyanate as described by Dakin (1910). It had a melting point of 178 to $180^{\circ} \mathrm{C}$. and an infrared spectrum identical with that recorded by Brown and Kies (1959).

A quantitative assessment of hydantoin-5-propionic acid excretion was made by comparing the size and intensity of the spots from varying quantities of urine with those of varying amounts of the pure acid after chromatography. Some of the test urines were similarly examined quantitatively for urocanic acid and formiminoglutamic acid excretion.

\section{RESULTS}

Table I shows the number of imidazole derivatives detected and their frequency. The excretion of hydantoin-5-propionic acid found in the two groups of subjects is summarized in Table II.

PRE-HISTIDINE CONTROL URINES No difference was found in the main between the subjects in the control group with regard to the number of imidazole derivatives detected, although their intensity was generally less in urine passed first thing in the morning. Imidazole lactic acid was usually detected in addition to the larger histidine spot (double in n-butanol/acetic acid/water). In the patients with anaemia due to folic acid-vitamin- $B_{12}$ deficiency an initially unidentified orange spot (using Pauly's reagent) was, however, found rather more often.

In the control urine hydantoin-5-propionic acid excretion was low in all those tested, little difference being found between either group. Only four patients with folic acid-vitamin- $B_{12}$ deficiency had an excretion obviously higher than the control group;
TABLE II

URINARY HYDANTOIN-5-PROPIONIC ACID EXCRETION M. MOLE/8 hourS-RANGE (MEAN)

\begin{tabular}{lcl} 
Urine & $\begin{array}{l}\text { Eleven Normal and } \\
\text { Anaemic Controls }\end{array}$ & $\begin{array}{l}\text { Forty Folic Acid and } \\
\text { Vitamin-B } \\
\text { Patients }\end{array}$ \\
\hline Control & $<0 \cdot 1-0 \cdot 2^{1}$ & $<0 \cdot 1-0 \cdot 2^{1}$ \\
Post-histidine & $0 \cdot 3-1 \cdot 0(0 \cdot 6)$ & $0 \cdot 3-10(4)$
\end{tabular}

${ }^{1}$ See text for four exceptions in folic acid-vitamin-B $\mathbf{B}_{12}$-deficient group.

in all these cases excretion increased to a higher level after histidine.

POST-HISTIDINE TEST URINES In all those tested, excretion of histidine and imidazole lactic acid was increased, the latter being no more prominent in either group of subjects. Imidazole acetic acid was detected rather more often in the controls than in the folic acid-vitamin- $\mathrm{B}_{12}$-deficient patients.

In many cases the eight-hour urine saves were split into three and five hours. These were usually pooled for testing, but in the few cases when they were examined separately excretion of imidazole lactic acid and imidazole acetic acid was more often highest in the first three hours. The eight-hour excretion of these two derivatives in the normal controls was about $0.3 \mathrm{~m}$. mole or $0.4 \%$ of the administered histidine.

A large proportion of the patients with folic acidvitamin- $B_{12}$ deficiency excreted urocanic acid and imidazole propionic acid, not infrequently in large amounts, while these compounds were just detected in one normal subject only. Urocanic acid was often more prominent, but in seven patients the excretion of these two acids appeared to be equally raised, and in nine the excretion of imidazole propionic acid exceeded urocanic acid; in seven of the latter urocanic acid was not detected.

The orange spot was just detected in the controls but became prominent in a large proportion of the folic acid-vitamin- $\mathrm{B}_{12}$-deficient patients. When urocanic acid and/or imidazole propionic acid

TABLE I

NUMBER OF SUBJECTS IN WHICH THE IMIDAZOLE WAS DETECTED

Imidazole

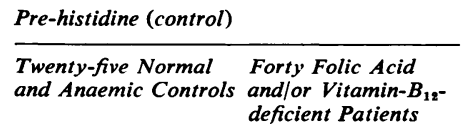

Post-histidine (test)

\begin{tabular}{ll}
\hline $\begin{array}{l}\text { Eleven Normal and } \\
\text { Anaemic Controls }\end{array}$ & $\begin{array}{l}\text { Forty Folic Acid } \\
\text { and/or Vitamin-B } \\
\text { deficient Patients }\end{array}$ \\
\hline
\end{tabular}

\section{Histidine}

Imidazole carboxylic acid

Imidazole lactic acid

Imidazole acetic acid

Orange spot urocanylglycine

Imidazole propionic acid

Urocanic acid

${ }^{1}$ Imidazole pyruvic acid was not detected in any of the urines. 
excretion was high, this orange spot was also pronounced, but excretion of the former two derivatives was most often greater.

All the normal and anaemic controls were Figlu negative while 30 of the folic acid-vitamin- $\mathrm{B}_{12}$ deficient patients were Figlu positive. Urocanic acid and/or imidazole-propionic acid excretion was increased in nine of the 10 Figlu-negative folic acidvitamin- $\mathrm{B}_{12}$-deficient patients, but in three of these urocanic acid was not detected. In three folic acidvitamin- $\mathrm{B}_{12}$-deficient patients, who were Figlu positive, urocanic acid and imidazole propionic acid were not detected; the orange spot, however, was prominent in two of them.

In 26 of the patients with folic acid-vitamin- $B_{12}$ deficiency the excretion of urocanic acid plus formiminoglutamic acid assessed quantitatively was between 0.1 and $2.2 \mathrm{~m}$.mole in the eight hours following histidine. In 12 of these patients urocanic acid excretion formed more than $50 \%$ of this total.

No obvious difference in excretory pattern was evident between patients with folic acid or vitamin$B_{12}$ deficiency alone, or combined.

Following histidine, hydantoin-5-propionic acid excretion increased moderately in all the normals and anaemic controls, with no obvious difference between them. The folic acid-vitamin- $B_{12}$-deficient patients, on the other hand, frequently had a marked increase. Thirty-two $(80 \%)$ of them excreted the abnormally high amount of more than $1.0 \mathrm{~m}$. mole in the eight hours following histidine. In one patient, who had rheumatoid arthritis and a low serum folic acid level, an abnormally high excretion of hydantoin5-propionic acid was the only index of abnormal histidine metabolism found. She also had a high urinary level of 4-aminoimidazole-5-carboxamide (Middleton, Coward, and Smith, 1964). No marked difference in excretion of hydantoin-5-propionic acid was found between the patients with single or combined folic acid-vitamin- $\mathrm{B}_{12}$ deficiency. Folic acid-vitamin- $\mathrm{B}_{12}$-deficient patients with an apparently normal excretion of hydantoin-5-propionic acid after histidine showed no other notable features to distinguish them from those with an abnormal level.

Both patients with psoriasis, who are not included in the Tables, were Figlu negative, but each showed an increased excretion of urocanic acid with imidazole propionic acid. One of them also had cirrhosis. Their serum folic acid and vitamin- $B_{12}$ levels were normal, as was their urinary excretion of hydantoin5-propionic acid and 4-aminoimidazole-5-carboxamide.

The orange spot had an $R f$ value of 35 to 39 ( $R$ urocanic acid 68) in n-butanol/acetic acid/water, similar to that of imidazole acetic acid (red-Pauly's reagent), but it could be distinguished from the latter in the other solvents. In all the solvents it ran as a discrete compact spot.

Separation of this unknown compound from the other imidazoles and partial purification was performed by elution from a paper chromatogram $\frac{\bar{\sigma}}{\mathrm{s}}$ followed by ion-exchange-column chromatography. $\vec{\nabla}$ Subsequent hydrolysis in $6 \mathrm{~N}$ hydrochloric acid $\varrho$ for 24 hours in a boiling water bath yielded urocanic $\tilde{\omega}$ acid, as assessed by paper chromatography, and the $\vec{\circ}$ orange spot disappeared. The unknown compound $\overrightarrow{-}$ had an absorption peak of $273 \mathrm{~m} \mu$ in $0.1 \mathrm{~N} \mathrm{HCl}, \vec{\omega}$ which after acid hydrolysis changed to $270 \mathrm{~m} \mu$, the same as that of urocanic acid. Alkaline hydrolysis? of urine, as performed in the urocanic acid method of $\vec{\infty}$ Bennett and Chanarin (1962), did not affect this ir orange spot, however. The above eluate contained $O$ no reducing substances either before or after hydrolysis. Amino acids were also absent from it before, but after acid hydrolysis glycine was detected. $\vec{c}$ An amino-acid conjugate of urocanic acid was also $\frac{\mathbb{D}}{2}$ suspected from the fact that the orange spot had $\mathbf{R f} \underset{\mathbb{D}}{\mathbb{D}}$ values higher than those of urocanic acid in phenol/ 3 ammonia/water, aqueous $\mathrm{KCl}$, and aqueous $\mathrm{NaCl} / \stackrel{\Phi}{-}$ acetic acid, but lower in the other solvents. The $\vec{\theta}$ pink-orange colour (bright gold in ultra-violet light) oे produced with the Altman reagent was also consistent with a glycine conjugate. Urocanylglycine was finally synthesized from urocanic acid and ethyl amino-acetate hydrochloride using dicyclohexyl- o carbodiimide after the procedure of Sheehan and $\%$ Hess (1955). This synthetic material co-chromato- $\triangle$ graphed exactly with the unknown spot in all the $\overline{\overrightarrow{0}}$ above solvents, and produced the same colours with $\exists$ the Pauly and Altman reagents.

\section{DISCUSSION}

The smallest amount of urocanic acid added to normal control urine which could be detected was $1 \mu \mathrm{g}$.; in two-seconds urine this represents an eighthour excretion of $0.11 \mathrm{~m} . \mathrm{mole}$. Since Chanarin, Bennett, and Berry (1962) have shown that the maximum excretion in the eight hours after a $15 \mathrm{~g}$. $\frac{\mathrm{O}}{\mathrm{O}}$ histidine oral load in normal subjects is $0.10 \mathrm{~m}$.mole, it was conjectured that examination of this $N$ quantity of urine would probably differentiate an $N$ abnormal from a normal response. The results bear out this original assumption.

The quantitative assessment of urocanic acid plus? formiminoglutamic acid excretion in this study agrees well with the data of Bennett and Chanarin (1962) who found on excretion of $0 \cdot 1$ to $10.0 \mathrm{~m}$. mole ${ }^{+}$ by similar patients in the eight hours following histidine.

Although all the metabolic pathways of histidine degradation have not yet been confirmed in man, the $\stackrel{\square}{\circ}$ 
likely course for the formation of the derivatives mentioned in this paper is shown in the Figure. Additional pathways in the metabolism of histamine are not shown. Since Schayer (1952) has shown that the histamine formed from histidine- $\mathrm{C}^{\mathbf{1 4}}$ in rats is excreted slowly over a number of days, the finding of excess excretion of histamine and its methylated derivatives would not have been expected in this study, as also that of the methylhistidines which are probably not derived directly from histidine.

Sen, McGeer, and Paul (1962) found imidazole propionic acid in normal human urine, and showed that in rats it was formed from both histidine and urocanic acid. Its increased excretion in deficiency of folic acid and vitamin- $\mathrm{B}_{12}$ recorded here suggests a similar pathway in man. Artefactual production from urocanic acid by electrolytic desalting, as noted by Žaníšek and Král (1953), was not responsible.

Imidazole carboxylic acid may also be derived from urocanic acid by analogy with phenolic acid metabolism. Booth, Emerson, Jones, and DeEds (1957) found that m-hydroxyhippuric acid, unlike the other metabolites, was not excreted until eight hours after caffeic acid dosage in man. The infrequent excretion of imidazole carboxylic acid within eight hours after histidine, observed here, may be comparable.

The finding of a glycine conjugate of urocanic acid in most of the subjects tested shows that this is a usual form of its excretion, although when large amounts of urocanic acid accumulate most is not excreted in this form.

Lower excretion in normal subjects of hydantoin5-propionic acid after histidine than that reported here was found by Brown et al. (1960); this may be the result of the different methods used. They also noted that urocanic acid plus formiminoglutamic acid excretion was less than that of hydantoin-5propionic acid. This is perhaps because it is not further metabolized. Imidazolone propionic acid is chemically very unstable and its detection in urine would not be expected.

Brown et al. (1960) concluded that the imidazole acetic acid excreted after histidine in normal men was produced from imidazole pyruvic acid. Imidazole lactic acid is no doubt similarly derived. The degree of interference with the main metabolic pathway of histidine by the deficiency of folic acid and vitamin- $\mathrm{B}_{12}$ in the patients studied here did not, however, result in an increased excretion of these compounds, and did not suggest that this alternative route is overactive in such conditions. The less frequent detection of imidazole acetic acid in these patients may indicate a further defect in them. Excretion as the riboside, which Kapeller-Adler and
Iggo (1957) have reported is not detectable by the diazo reaction, may have been the explanation in some instances, although Brown et al. (1960) found that the excretion of this conjugate was greater than that of the free acid after histidine in normal subjects. The latter authors also noted that imidazole acetic acid excretion was highest in the first hour after histidine, and it can be concluded that intestinal bacterial fermentation played no part in its production. Imidazole pyruvic acid itself was perhaps not detected because of its known lability. No special precaution was taken to preserve it. It also yields only a faint colour with Pauly's reagent.

These normally minor metabolic products have been shown by a number of authors (see Holton, Lewis, and Moore, 1964) to be excreted in excess by patients lacking in histidase, when conversion of histidine to urocanic acid is blocked.

Bennett and Chanarin (1961) and Knowles (1961) postulated that the excretion of urocanic acid in deficiency of folic acid and vitamin- $B_{12}$ results from suppressed formation or inhibition of urocanase by accumulated formiminoglutamic acid. Decreased urocanase activity, however, occurs in diseases other than deficiency of folic acid and vitamin $\mathbf{B}_{12}$. Thus Knowles, Shaldon, and Fleming (1963) found abnormal Figlu tests in patients with liver disease, which were not corrected by folic acid therapy, and the presence of significant amounts of urocanic acid in the urine of patients with kwashiorkor, reported by Whitehead and Arnstein (1961), has also been suggested by Whitehead (1964) to be unassociated with deficiency of folic acid and vitamin $\mathrm{B}_{12}$. Although positive Figlu tests in patients with various dermatoses, which reverted to normal with folic acid treatment, have been reported by Knowles, Shuster, and Wells (1963), there was no evidence that the two patients with psoriasis reported here were deficient in folic acid and vitamin $B_{12}$. In one of them cirrhosis may have been the cause of the increased urocanic acid excretion. On the other hand since Schwarz (1961) demonstrated that guinea-pig epidermis can produce urocanic acid from histidine, the high cellular activity of this tissue in psoriasis may have contributed to the findings here.

The present study has shown that examination for histidine metabolites other than formiminoglutamic acid in the Figlu test is necessary to confirm the diagnosis in every case of deficiency of folic acid and vitamin $B_{12}$. This complex task can be performed most simply by paper chromatography. The above considerations, however, emphasize that such findings are not diagnostic of these deficiencies alone.

I am grateful to the clinicians of the Southampton group of hospitals for the opportunity to investigate the patients 
under their care, to Dr. S. C. Dobson, in whose department the haematological investigations were performed, for helpful cooperation, and to Miss Jane M. Beavon (1963) who performed the serum folic acid and vitamin$B_{12}$ estimations. I also wish to thank Dr. S. Kasemsri and the technicians of my Department for help with some of the investigations, and Professor K. A. Munday, of the Physiology and Biochemistry Department, Southampton University, for the infrared analysis.

\section{REFERENCES}

Beavon, J. M. (1963). J. med. Lab. Tech., 20, 183.

Bennett, M. C., and Chanarin, I. (1961). Lancet, 2, 1095.

- , (1962). Nature (Lond.), 196, 271.

Bergmann, F., and Dikstein, I. (1958). Meth. biochem. Anal., 6, p. 88.

Booth, A. N., Emerson, O. H., Jones, F. T., and DeEds, F. (1957). J. biol. Chem., 229, 51.

Brown, D. D., and Kies, M. W. (1959). Ibid., 234, 3182.

-, Silva, O. L., McDonald, P. B., Snyder, S. H., and Kies, M. W. (1960). Ibid., 235, 154.

Chanarin, I., Bennett, M. C., and Berry, V. (1962). J. clin. Path., 15, 269.
Dagnall, P. (1962). Lancet, 1, 1408.

Dakin, H. D. (1910). Amer. chem. J., 44, 48.

Holton, J. B., Lewis, F. J. W., and Moore, G. R. (1964). J. clin. Path., 17,671 .

Hubbard, R. W., and Block, W. D. (1962). Clin. Chem., 8, 424.

Kapeller-Adler, R., and Iggo, B. (1957). Biochim. biophys. Acta (Amst.), 25, 394.

Knowles, J. P. (1961). Lancet, 2, 1149.

Shaldon, S., and Fleming, A. (1963). Clin. Sci., 24, 39.

Shuster, S., and Wells, G. C. (1963). Lancet, 1, 1138.

McGeer, E. G., Robertson, M. C., and McGeer, P. L. (1961). Canad. J. Biochem., 39, 605 .

Middleton, J. E. Coward, R. F and Smith, P. (1964). Lancet, 2, 258.

Schayer, R. W. (1952). J. biol. Chem., 199, 245.

Schwarz, E. (1961). Biochem. Z., 334, 415.

Sen, N. P., McGeer, P. L., and Paul, R. M. (1962). Biochem. biophys. Res. Commun., 9, 257.

Sheehan, J. C., and Hess, G. P. (1955). J. Amer. chem. Soc., 77, 1067.

Smith, I., and Birchenough, M. (1960). In Chromatographic and Electrophoretic Techniques, edited by I. Smith, vol. 1, p. 212. Heinemann, London.

Whitehead, R. G. (1964). Clin. Sci., 26, 271.

, and Arnstein, H. R. V. (1961). Nature (Lond.), 190, 1105.

Žaníšek, A., and Král, J. A. (1953). Biochim. biophys. Acta (Amst.), 12, 479. 